\title{
ASYMPTOTIC BEHAVIOR OF ENTIRE FUNCTIONS OF IMPROVED REGULAR GROWTH IN THE METRIC OF $L^{p}[0,2 \pi]$
}

\begin{abstract}
We describe an asymptotic behavior of entire functions of improved regular growth with zeros on a finite system of rays in the metric of $L^{p}[0,2 \pi]$.

Key words and phrases: entire function of improved regular growth, finite system of rays, Fourier coefficients, Hausdorff-Young theorem.
\end{abstract}

Ivan Franko State Pedagogical University, 24 Franka str., 82100, Drohobych, Ukraine

E-mail: khats@ukr.net

\section{INTRODUCTION AND MAIN RESULT}

The asymptotic behavior of entire and meromorphic functions of positive order of completely regular growth (for details, see $[2,3,5,11]$ ) in the metric of $L^{p}[0,2 \pi]$ was described in $[6,11,12,13]$. Similar results for entire functions of zero order whose zero-counting functions are slowly increasing were obtained in [1]. In particular, from [11, Theorem 7.2, p. 78] it follows the following statement.

Theorem A. If an entire function $f$ of order $\rho \in(0,+\infty)$ with the indicator $h(\theta, f)$ is of completely regular growth, then for any $p \in[1,+\infty)$

$$
\lim _{r \rightarrow+\infty}\left\{\frac{1}{2 \pi} \int_{0}^{2 \pi}\left|\frac{\log \left|f\left(r e^{i \theta}\right)\right|}{r^{\rho}}-h(\theta, f)\right|^{p} d \theta\right\}^{1 / p}=0 .
$$

Conversely, if for some entire function $f, f(0)=1$, there exist $p \in[1,+\infty)$ and $\widetilde{h} \in L^{p}[0,2 \pi]$ such that

$$
\lim _{r \rightarrow+\infty}\left\{\frac{1}{2 \pi} \int_{0}^{2 \pi}\left|\frac{\log \left|f\left(r e^{i \theta}\right)\right|}{r^{\rho}}-\widetilde{h}(\theta)\right|^{p} d \theta\right\}^{1 / p}=0,
$$

then $f$ is of completely regular growth and $\widetilde{h}(\theta)=h(\theta, f)$ for almost all $\theta \in[0,2 \pi]$.

The aim of the present paper is obtain an analog of Theorem A for entire functions of improved regular growth with zeros on a finite system of rays (see $[4,7,8,9,10,14]$ ).

An entire function $f$ is called a function of improved regular growth (see $[7,8,9,10,14])$ if for some $\rho \in(0,+\infty)$ and $\rho_{1} \in(0, \rho)$, and a $2 \pi$-periodic $\rho$-trigonometrically convex function $h(\varphi) \not \equiv-\infty$ there exists the set $U \subset \mathbb{C}$ contained in the union of disks with finite sum of radii and such that $\log |f(z)|=|z|^{\rho} h(\varphi)+o\left(|z|^{\rho_{1}}\right), U \not \supset z=r e^{i \varphi} \rightarrow \infty$.

If an entire function $f$ is a function of improved regular growth, then it has the order $\rho$ and indicator $h$ [14]. Our main result is the following theorem. 
Theorem 1. An entire function $f$ of order $\rho \in(0,+\infty)$ with zeros on a finite system of rays $\left\{z: \arg z=\psi_{j}\right\}, j \in\{1, \ldots, m\}, 0 \leq \psi_{1}<\psi_{2}<\ldots<\psi_{m}<2 \pi$, is a function of improved regular growth if and only if for some $\rho_{2} \in(0, \rho)$ and any $p \in[1,+\infty)$, one has

$$
\left\{\frac{1}{2 \pi} \int_{0}^{2 \pi}\left|\frac{\log \left|f\left(r e^{i \varphi}\right)\right|}{r^{\rho}}-h(\varphi)\right|^{p} d \varphi\right\}^{1 / p}=o\left(r^{\rho_{2}-\rho}\right), \quad r \rightarrow+\infty .
$$

\section{PRELIMINARIES}

Let $f$ be an entire function with $f(0)=1$, let $\left(\lambda_{n}\right)_{n \in \mathbb{N}}$ be the sequence of its zeros, let $p$ be the smallest integer for which $\sum_{n=1}^{\infty}\left|\lambda_{n}\right|^{-p-1}<+\infty$, let $Q_{\rho}$ be the coefficient of $z^{\rho}$ in the exponential factor in the Hadamard-Borel representation [5, p. 38] of an entire function of finite order, and let $c_{k}(r, \log |f|)$ be the Fourier coefficients of $\log |f|$, i.e.

$$
c_{k}(r, \log |f|):=\frac{1}{2 \pi} \int_{0}^{2 \pi} e^{-i k \varphi} \log \left|f\left(r e^{i \varphi}\right)\right| d \varphi, \quad k \in \mathbb{Z}, \quad r>0 .
$$

Further, let $f$ be an entire function of order $\rho \in(0,+\infty)$ with zeros on a finite system of rays $\left\{z: \arg z=\psi_{j}\right\}, j \in\{1, \ldots, m\}, 0 \leq \psi_{1}<\psi_{2}<\ldots<\psi_{m}<2 \pi$. Furthermore, if $\rho$ is noninteger and $f$ is a function of improved regular growth, then an indicator $h$ of $f$ has the form $([8,10,14])$

$$
h(\varphi)=\sum_{j=1}^{m} h_{j}(\varphi)
$$

where $h_{j}(\varphi)$ is a $2 \pi$-periodic function such that on $\left[\psi_{j}, \psi_{j}+2 \pi\right)$

$$
h_{j}(\varphi)=\frac{\pi \Delta_{j}}{\sin \pi \rho} \cos \rho\left(\varphi-\psi_{j}-\pi\right), \quad \Delta_{j} \in[0,+\infty)
$$

In the case $\rho \in \mathbb{N}$, the indicator $h$ is defined by the formula $([8,9,10])$

$$
h(\varphi)=\left\{\begin{array}{l}
\tau_{f} \cos \left(\rho \varphi+\theta_{f}\right)+\sum_{j=1}^{m} h_{j}(\varphi), \quad p=\rho, \\
Q_{\rho} \cos \rho \varphi, \quad p=\rho-1,
\end{array}\right.
$$

where $\delta_{f} \in \mathbb{C}, \tau_{f}=\left|\delta_{f} / \rho+Q_{\rho}\right|, \theta_{f}=\arg \left(\delta_{f} / \rho+Q_{\rho}\right)$ and $h_{j}(\varphi)$ is a $2 \pi$-periodic function such that on $\left[\psi_{j}, \psi_{j}+2 \pi\right)$ we have

$$
h_{j}(\varphi)=\Delta_{j}\left(\pi-\varphi+\psi_{j}\right) \sin \rho\left(\varphi-\psi_{j}\right)-\frac{\Delta_{j}}{\rho} \cos \rho\left(\varphi-\psi_{j}\right) .
$$

Lemma 1 ([8]). If an entire function $f$ of order $\rho \in(0,+\infty)$ with zeros on a finite system of rays $\left\{z: \arg z=\psi_{j}\right\}, j \in\{1, \ldots, m\}, 0 \leq \psi_{1}<\psi_{2}<\ldots<\psi_{m}<2 \pi$, is of improved regular growth, then for some $\rho_{3} \in(0, \rho)$

$$
c_{k}(r, \log |f|)=c_{k} r^{\rho}+\frac{o\left(r^{\rho_{3}}\right)}{k^{2}+1}, \quad r \rightarrow+\infty,
$$

holds uniformly in $k \in \mathbb{Z}$, where

$$
c_{k}:=\frac{1}{2 \pi} \int_{0}^{2 \pi} e^{-i k \varphi} h(\varphi) d \varphi=\frac{\rho}{\rho^{2}-k^{2}} \sum_{j=1}^{m} \Delta_{j} e^{-i k \psi_{j}}, \quad \Delta_{j} \in[0,+\infty),
$$


for a noninteger $\rho$, and

$$
c_{k}=\left\{\begin{array}{l}
\frac{\rho}{\rho^{2}-k^{2}} \sum_{j=1}^{m} \Delta_{j} e^{-i k \psi_{j}}, \quad|k| \neq \rho=p, \\
\frac{\tau_{f} e^{i \theta_{f}}}{2}-\frac{1}{4 \rho} \sum_{j=1}^{m} \Delta_{j} e^{-i \rho \psi_{j}}, \quad k=\rho=p, \\
0, \quad|k| \neq \rho=p+1, \\
\frac{Q_{\rho}}{2}, \quad k=\rho=p+1,
\end{array}\right.
$$

if $\rho$ is an integer.

Remark that, using Lemma 1 and the Riesz-Fischer theorem [11, p. 5], we get that there exists an indicator $h \in L^{2}[0,2 \pi]$ defined by the equality $h(\varphi):=\sum_{k \in \mathbb{Z}} c_{k} e^{i k \varphi}$ (see [11, Definition 7.2, p. 77]).

\section{ProOF OF THEOREM 1}

Necessity. If an entire function $f$ of order $\rho \in(0,+\infty)$ with zeros on a finite system of rays $\left\{z: \arg z=\psi_{j}\right\}, j \in\{1, \ldots, m\}, 0 \leq \psi_{1}<\psi_{2}<\ldots<\psi_{m}<2 \pi$, is of improved regular growth, then by (2), we have

$$
\left|\frac{c_{k}(r, \log |f|)}{r^{\rho}}-c_{k}\right| \leq \frac{C}{k^{2}+1}, \quad k \in \mathbb{Z},
$$

for some constant $C>0$ and all $r>0$. In view of this, the sequence $\left(r^{-} \rho_{c_{k}}(r, \log |f|)-c_{k}\right)_{k \in \mathbb{Z}}$ belongs to the space $l_{q}$ for all $q>1$ and $r>0$. Moreover, applying the Hausdorff-Young theorem [11, p. 5], for $p \geq 2, p^{-1}+q^{-1}=1$, we get

$$
\left\{\frac{1}{2 \pi} \int_{0}^{2 \pi}\left|\frac{\log \left|f\left(r e^{i \varphi}\right)\right|}{r^{\rho}}-h(\varphi)\right|^{p} d \varphi\right\}^{1 / p} \leq\left\{\sum_{k \in \mathbb{Z}}\left|\frac{c_{k}(r, \log |f|)}{r^{\rho}}-c_{k}\right|^{q}\right\}^{1 / q} .
$$

According to (3), the last series is uniformly convergent with respect to $r$. Therefore, using Lemma 1, we obtain (1) for $p \geq 2$. From this and Hölder's inequality it follows that (1) holds for $1 \leq p<2$.

Sufficiency. Let (1) holds. Then for some $\rho_{2} \in(0, \rho)$ and each $k \in \mathbb{Z}$

$$
\begin{aligned}
\left|\frac{c_{k}(r, \log |f|)}{r^{\rho}}-c_{k}\right| & \leq \frac{1}{2 \pi} \int_{0}^{2 \pi}\left|\frac{\log \left|f\left(r e^{i \varphi}\right)\right|}{r^{\rho}}-h(\varphi)\right| d \varphi \\
& \leq\left\{\frac{1}{2 \pi} \int_{0}^{2 \pi}\left|\frac{\log \left|f\left(r e^{i \varphi}\right)\right|}{r^{\rho}}-h(\varphi)\right|^{p} d \varphi\right\}^{1 / p}=o\left(r^{\rho_{2}-\rho}\right), \quad r \rightarrow+\infty
\end{aligned}
$$

Hence, for some $\rho_{2} \in(0, \rho)$ and each $k \in \mathbb{Z}$ we get $c_{k}(r, \log |f|)=c_{k} r^{\rho}+o\left(r^{\rho_{2}}\right), r \rightarrow+\infty$. Then by [10, Theorem 1, p. 1718] an entire function $f$ is a function of improved regular growth. This concludes the proof of the theorem. 


\section{REFERENCES}

[1] Bodnar O.V., Zabolots'kyi M.V. Criteria for the regularity of growth of the logarithm of modulus and the argument of an entire function. Ukrainian Math. J. 2010, 62 (7), 1028-1039. doi:10.1007/s11253-010-0411-x (translation of Ukr. Mat. Zh. 2010, 62 (7), 885-893. (in Ukrainian))

[2] Gol'dberg A.A. B.Ya. Levin, the founder of the theory of entire functions of completely regular growth. Mat. Fiz., Anal., Geom. 1994, 1 (2), 186-192. (in Russian)

[3] Gol'dberg A.A., Levin B.Ya., Ostrovskii I.V. Entire and meromorphic functions. VINITI 1991, 85, 5-186. (in Russian)

[4] Hirnyk M.O. Subharmonic functions of improved regular growth. Dopov. Nats. Akad. Nauk Ukr. 2009, 4, 13-18. (in Ukrainian)

[5] Levin B.Ya. Distribution of zeros of entire functions. Gostekhizdat, Moscow, 1956. (in Russian)

[6] Kalynets' R.Z., Kondratyuk A.A. On the regularity of the growth of the modulus and argument of an entire function in the metric of $L^{p}[0,2 \pi]$. Ukrainian Math. J. 1998, 50 (7), 1009-1018. doi: 10.1007/BF02528830 (translation of Ukr. Mat. Zh. 1998, 50 (7), 889-896. (in Ukrainian))

[7] Khats' R.V. Asymptotic behavior of averaging of entire functions of improved regular growth. Carpathian Math. Publ. 2013, 5 (1), 129-133.

[8] Khats' R.V. Averaging of entire functions of improved regular growth with zeros on a finite system of rays. Visn. Nats. Univ. L'viv. Politekh., Fiz.-Mat. Nauky. 2011, 718 (718), 10-14.

[9] Khats' R.V. On entire functions of improved regular growth of integer order with zeros on a finite system of rays. Mat. Stud. 2006, 26 (1), 17-24.

[10] Khats' R.V. Regularity of growth of Fourier coefficients of entire functions of improved regular growth. Ukrainian Math. J. 2012, 63 (12), 1953-1960. doi:10.1007/s11253-012-0624-2 (translation of Ukr. Mat. Zh. 2011,63 (12), 1717-1723. (in Ukrainian))

[11] Kondratyuk A.A. Fourier series and meromorphic functions. Vyshcha shkola, Kyiv, 1988. (in Russian)

[12] Vasyl'kiv Ya.V. Asymptotic behavior of logarithmic derivatives and logarithms of meromorphic functions of completely regular growth in the metric of $L^{p}[0,2 \pi]$. I. Mat. Stud. 1999, 12 (1), 37-58. (in Ukrainian)

[13] Vasyl'kiv Ya.V. Asymptotic behavior of logarithmic derivatives and logarithms of meromorphic functions of completely regular growth in the metric of $L^{p}[0,2 \pi]$. II. Mat. Stud. 1999, 12 (2), 135-144. (in Ukrainian)

[14] Vynnyts'kyi B.V., Khats' R.V. On growth regularity of entire function of noninteger order with zeros on a finite system of rays. Mat. Stud. 2005, 24 (1), 31-38. (in Ukrainian)

Received 21.08.2013

Хаць Р.В. Асимптотична поведінка иуілих функцій покращеного регулярного зростання в $L^{p}[0,2 \pi]$ метриці // Карпатські математичні публікації. - 2013. - Т.5, №2. - С. 341-344.

Описано асимптотичну поведінку цілих функцій покращеного регулярного зростання 3 нулями на скінченній системі променів в $L^{p}[0,2 \pi]$-метриці.

Ключові слова і фрази: ціла функція покращеного регулярного зростання, скінченна система променів, коефіцієнти Фур'є, теорема Гаусдорфа-Юнга.

Хаць Р.В. Асимптотическое поведение цельх функиий улучиенного регулярного роста в $L^{p}[0,2 \pi]$ метрике // Карпатские математические публикации. - 2013. — Т.5, №2. - С. 341-344.

Описано асимптотическое поведение целых функций улучшенного регулярного роста с нулями на конечной системе лучей в $L^{p}[0,2 \pi]$-метрике.

Ключевые слова и фразы: целая функция улучшенного регулярного роста, конечная система лучей, коэффициенты Фурье, теорема Гаусдорфа-Юнга. 